\title{
Vídeo educativo y rendimiento académico en la enseñanza superior a distancia
}

\section{Educational video and academic performance in higher distance education}

\author{
Damián de la Fuente Sánchez \\ Montserrat Hernández Solís \\ Inmaculada Pra Martos \\ Universidad Nacional de Educación a Distancia, UNED (España)
}

\section{Resumen}

En los últimos años el desarrollo de las tecnologías de la información y el conocimiento han tenido un notable impacto en la enseñanza universitaria a distancia, tanto a nivel cuantitativo como cualitativo. Se ha observado que, a pesar de la aparición de numerosos y novedosos recursos didácticos, el vídeo, en su formato convencional o incorporando la interactividad, sigue siendo todavía el preferido por profesores y alumnos para mejorar la calidad del proceso de aprendizaje en materias económicas de naturaleza cuantitativa. El objetivo central de esta investigación es contribuir al aumento del escaso número de trabajos publicados en nuestro país sobre los efectos que tienen los recursos educativos en formato vídeo (estándar e interactivo) sobre la mejora del rendimiento académico en un entorno metodológico de enseñanza universitaria a distancia. El análisis se ha centrado en una asignatura percibida como de especial dificultad por los estudiantes, tal cual es la matemática financiera que se programa en el plan de estudios del Grado en Administración y Dirección de Empresas de la Universidad Nacional de Educación a Distancia (UNED). A lo largo de tres cursos académicos consecutivos se fueron recopilando mediante una encuesta los datos necesarios de una muestra suficientemente representativa para comprobar, mediante distintas técnicas estadísticas, la relación positiva del uso de estos recursos sobre el rendimiento académico, la utilidad global que les proporcionó a los estudiantes que los utilizaron y el valor añadido que les reportó frente a otras alternativas tradicionales de estudio como son los materiales escritos (textos y guías didácticas).

Palabras clave: enseñanza superior; ciencias económicas; medida del rendimiento; método multimedia; rendimiento comparado; universidad a distancia.

\begin{abstract}
In the last years the development of the information and communication technologies has had a significant impact on the university distance learning, both in a quantitative and in a qualitative manner. It has been observed that, despite the appearance of numerous and novel didactic resources, video, in its conventional format or incorporating interactivity, is still preferred by teachers and students to improve the quality of learning process in economic
\end{abstract}


quantitative subjects. The goal of this research is to contribute to the increase of the small number of papers which have been published in our country about the way in which the effects of educational resources in video format (standard and interactive) influence on improving learning in a distance learning university environment. The analysis has been focused on a subject perceived as particularly difficult for students, called Financial Mathematics, which is studied in the Administration and Management Degree, Distance Learning National University (UNED). Over three consecutive academic courses a sufficient representative sample was collected through a sample to verify, by using different statistics techniques, a positive relationship between the use of these resources over the academic performance, the global utility that these resources provided to the students who used them and the added value that these resources presented to them, over other traditional alternatives to study, such as written materials (texts and teaching guides).

Keywords: higher education; economics; achievement measurement; multimedia method; comparative achievement; open university.

Nadie duda que la evolución de la tecnología ha provocado cambios sustanciales en las sociedades en las que se han desarrollado (Cabero, Llorente y Román, 2007). En este contexto de continuas modificaciones, las tecnologías de la información y la comunicación (TIC) no han sido ajenos a estos fenómenos de transformación en diversos ámbitos, incluido, por supuesto, el de la educación y el aprendizaje. Muchos autores han señalado a lo largo de los últimos años la utilidad de las TIC en los entornos de enseñanza y aprendizaje (Castaño-Muñoz, Durant y Teresa, 2015; Estévez y García, 2015), tales como la ampliación de la oferta formativa e informativa, la apertura de nuevas posibilidades de la tutorización, la eliminación de barreras de espacio y de tiempo, la facilitación del trabajo personal, del trabajo colaborativo y del autoaprendizaje, además de potenciar la interactividad y la flexibilidad en el aprendizaje.

La educación superior a distancia (o mejor la educación superior semipresencial) no ha sido ajena al desarrollo de estas tecnologías y, en consecuencia, ha tenido que afrontar el reto de transformar la clase presencial en forma de tutoría a la clase virtual a partir, sobre todo, del uso de diversas herramientas multimedia y de nuevos recursos didácticos distintos a los materiales escritos tradicionales (textos y guías de estudio). En esta evolución hacia la docencia en línea o educación digital ha sido fundamental el desarrollo de internet y su capacidad para integrar un conjunto de materiales multimedia de diversa naturaleza (webconferencias, videoconferencias, chats, videos y audios, mini libros electrónicos o laboratorios remotos).

Ese amplio catálogo de recursos se ha recogido eintegrado en plataformas virtuales digitales con un doble objetivo, tal como señalan Imbernón, Silva y Guzmán (2011): por un lado, la consolidación y la potenciación de acciones formativas de amplio recorrido y, por otro lado, la creación de espacios de comunicación e intercambio de opiniones e ideas entre los propios estudiantes y entre ellos y el profesorado. 
Los recursos multimedia, que se han ido incorporando paulatinamente en la facilitación del proceso de aprendizaje de materias de tipo cuantitativo económico, han sido muy variados en el pasado más reciente: clips de películas para ilustrar diversos conceptos económicos (Leet y House, 2003; Mateer y Li, 2008; Sexton, 2006; Rodríguez, 2011), vídeos o audios (Siegfried et al., 1991), ilustraciones de novelas (Vachis y Bohanan, 2012), novelas (Cotti y Marianne, 2012), música (Tinari y Khandke, 2000), espectáculos y series de televisión (Hall, 2005; Lucasen y Thomas, 2010; Mateer y Lee, 2008) e incluso las redes sociales más populares (Al-Bahrani y Darshak, 2015).

Entre todos esos recursos, el vídeo ha sido uno de los más utilizados, tanto en la enseñanza presencial como en la modalidad a distancia. Especialmente en los últimos años es significativo el incremento en el uso de esta tecnología, tal como se observa en los repositorios de videos en las academias virtuales (Coursera, Academia Khan, Miriada X, OpenCourseWare, etc.) o en el surgimiento de lo que se ha denominado cursos en línea, masivos y abiertos (los llamados MOOCs en términos anglosajones).

No vamos a ocupar mucho espacio en señalar sus bondades a la hora de canalizar la información y el conocimiento y constituirse en un fuerte instrumento motivador de los estudiantes porque no es ese el objetivo de esta investigación. Nos centraremos más bien en su eficacia para conseguir determinados objetivos de tipo académico a partir de diversos estudios a nivel internacional: desde el aumento de las competencias comunicativas de los alumnos a la hora de relacionarse entre ellos y con el profesor (Borup, West y Graham, 2013) hasta la mejora de las relaciones entre profesor y alumno (Yanghee y Thayne, 2015), pasando por una mayor motivación de los alumnos (Hee y Scott, 2005; Ljubojevic et al., 2014; Bravo et al., 2011), la mejora de los hábitos de estudio (Dickie, 2009), o mayores niveles de reflexión y pensamiento crítico (Hilgenberg y Tolone, 2000; Leijen et al., 2008). Sin embargo, son escasos los trabajos que analizan estas cuestiones en nuestro país, especialmente en el ámbito de la enseñanza universitaria a distancia o semipresencial.

En este trabajo se pretende analizar el efecto que ha tenido la utilización de diversos recursos multimedia sobre el rendimiento académico final obtenido en una asignatura de tipo cuantitativo dentro del plan de estudios del Grado en Administración y Dirección de Empresas (ADE) de la Universidad Nacional de Educación a Distancia (UNED) en España. En nuestro estudio comprobaremos si existe una relación positiva entre el uso del video en distintos formatos y el rendimiento académico medido a través de la calificación obtenida en una prueba presencial, tal como confirman un buen número de trabajos en la literatura extranjera: Hsin y Cigas (2013), Wieling y Hofman (2010), Stiubiener et al. (2012), Berk (2009), Boster y otros (2006, 2007), Griffin, Mitchel y Thompson (2009), Zhang et al. (2006) y Vajoczki et al. (2010), o por el contrario, el impacto de la utilización del video es pequeño o incluso nulo: Bennett y Glover (2008), Boster y otros (2006), Dupagne, Millette y Grinfeder (2009), Hill y Nelson (2011), Mbarika et al. (2000), O’ Bannon et al. (2011) y Ross y Bell (2007). 
También se analizará la relación entre el rendimiento académico y una serie de variables independientes relacionadas con los hábitos de estudio y, por último, se comprobará la influencia que pudieran tener otras variables relacionadas con el diseño y la usabilidad de los recursos sobre el valor añadido que les reporta a los alumnos su uso frente a otras alternativas de estudio más tradicionales.

Dada la naturaleza de los datos obtenidos a través de las encuestas realizadas y las posibilidades que ofrece su tratamiento, hemos considerado diversos objetivos a alcanzar en esta investigación:

- En primer lugar, comparar el rendimiento académico medido por la calificación obtenida en la prueba presencial escrita (que en esta universidad es obligatoria y se realiza en la convocatoria ordinaria de febrero/junio o extraordinaria de septiembre) entre los alumnos que utilizaron los recursos multimedia y los que optaron por no hacer uso de ellos.

- En segundo lugar, comprobar si existe una relación positiva entre el rendimiento académico y la utilidad global que ha proporcionado a los alumnos la consulta de esos recursos multimedia.

- En tercer lugar, analizar el efecto de otro tipo de variables (horas de trabajo semanal, nivel previo de matemáticas, consulta de recursos multimedia) sobre dicho rendimiento.

- Por último, contrastar si los estudiantes tienen una percepción positiva de la utilidad de estos recursos multimedia en su proceso de aprendizaje.

\section{MATERIAL Y MÉTODOS}

Los recursos multimedia a los que haremos referencia, en este trabajo, son de tres tipos: mini videos conceptuales interactivos, grabaciones convencionales de sesiones de tutoría relativas a conceptos teóricos del temario y de resolución de ejercicios prácticos. En el primer caso, se trata de vídeos educativos en la línea señalada por Pascual (2011), es decir, un medio didáctico que facilita el desarrollo del proceso de aprendizaje con unas características muy definidas en cuanto a su naturaleza. Por tanto, podríamos definir a esta herramienta como un video de corta duración cuyo objetivo es reforzar y consolidar el aprendizaje de ciertos conceptos de la materia. No hay que confundir un mini video con un video convencional. Las diferencias más importantes son las siguientes: la duración, el formato y el papel que juega el alumno en el proceso de aprendizaje a través de esta herramienta. En cuanto a la duración, el mini video explica un concepto y desarrolla alguna demostración o razonamiento matemático en un breve espacio de tiempo, que conviene no sea superior a cinco minutos. La segunda diferencia tiene que ver con el diseño del formato empleado en el mini video puesto que se utilizan presentaciones minimalistas en las que se sugiere una frase, gráfico o una ecuación. Se establece así una base prácticamente en blanco sobre la que se desarrollan las explicaciones de cada mini video y que, 
posteriormente, los estudiantes pueden descargar para que sirvan como soporte de cara al estudio y reflexión posterior. La tercera característica diferenciadora del mini video es el rol que desempeña el estudiante. Para reducir el grado de pasividad que, por su propia naturaleza, presenta este formato, se ha introducido la interactividad. Con la ayuda de determinadas aplicaciones y plataformas (educanon, tdex, etc.), se han introducido dentro de cada vídeo convencional una batería de preguntas cerradas de elección múltiple o abiertas sobre el contenido que se va visualizando, de tal forma que el vídeo no avanza si el estudiante no contesta. Si la respuesta señalada es la correcta, el vídeo continúa hasta que vuelve a pararse en la siguiente pregunta. Si la respuesta elegida no es la correcta, se señala por qué no lo es y se indica cuál debería haber sido la seleccionada.

Figura 1. Mini video interactivo con retroalimentación

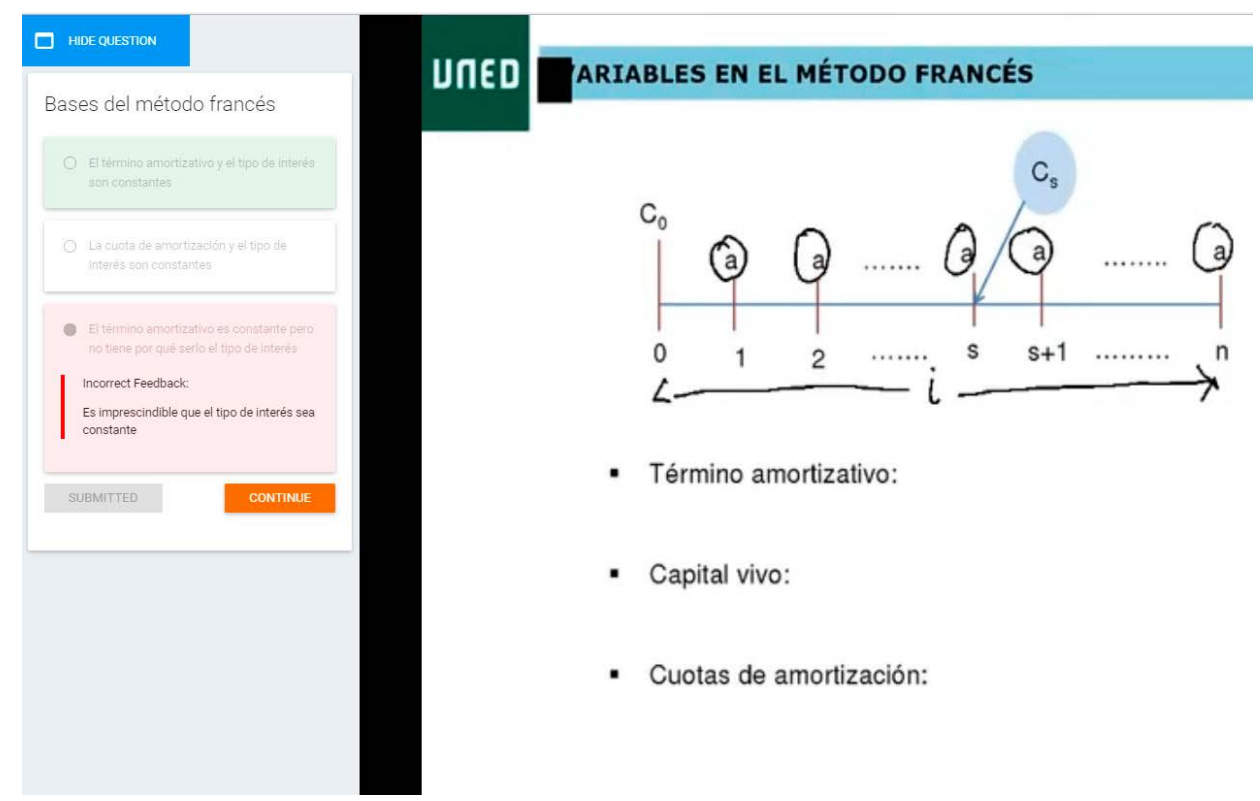

Las grabaciones de sesiones de tutorías se refieren a las clases presenciales impartidas por el coordinador de la asignatura, durante el curso académico correspondiente, en el Centro Asociado de la UNED Madrid-Giner de los Ríos. Por último, las grabaciones de ejercicios resueltos se centran en aspectos prácticos de los contenidos de la asignatura. En estos dos últimos formatos, se suprime la interactividad de los mini videos y se amplía la duración puesto que se trata de tutorías y clases magistrales sobre los contenidos de cada capítulo de la materia. 
Figura 2. Grabación clase teoría

\section{UกED}

\section{TEMA 4: LEYES FINANCIERAS DE DESCUENTO}

$$
\begin{gathered}
\begin{array}{c}
\text { VALOR DESCONTADO Y } \\
\text { DESCUENTO }
\end{array} \\
V_{0}=C \cdot A_{2}(z)=\frac{C}{1+i \cdot z} \\
D=C-V_{0}=C-\frac{C}{1+i \cdot z}=\frac{C \cdot i \cdot z}{1+i \cdot z} \\
\text { TIPOS DE DESCUENTO } \\
\text { EQUIVALENTES }
\end{gathered}
$$

Teniendo en cuenta que el parámetro "i" que aparece en la expresión de la ley de descuento racional es el mismo que el que aparece en la de capitalización simple, la obtención de los tantos equivalentes sigue el mismo procedimiento $y$, por tanto se verifica que:

$$
i=i_{m} \cdot m ; i_{m}=\frac{i}{m}
$$

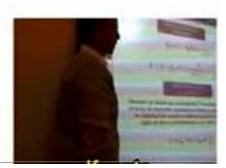

A la hora de producir los mini vídeos, se pueden seguir distintas orientaciones y recomendaciones en las que se señalan diferentes etapas y se proponen diversas variables pedagógicas y de comunicación (Martínez, 2004; Sigüenza, 2004). En nuestro caso, esas fases se han concretado en la identificación de los conceptos a tratar, la preparación del guion, la elaboración de los esquemas y/o transparencias y, por último, el ensayo previo y la grabación. El resultado final ha sido un vídeo de corta duración (en torno a cinco minutos) para mantener al máximo la concentración del estudiante y evitar, en la medida de lo posible, la pérdida de interés (tal como señalan Ellis y Childs, 1999; Wallace, 2003).

Todo este material se ha realizado utilizando la tecnología AVIP (audio y vídeo vía IP) que la UNED ha desarrollado en el marco de un ambicioso plan de desarrollo tecnológico. Más concretamente, se ha aprovechado la denominada conferencia en línea para elaborar vídeos y grabaciones de tutorías que los alumnos pueden descargar en formato comprimido mp4 o visualizarlos vía streaming con la única limitación de su ancho de banda en la conexión a internet. 
Con el fin de obtener el máximo provecho de estos recursos, se planteó a los alumnos una estrategia de uso, antes, durante y después del visionado, a partir de las recomendaciones de Cabero, Llorente y Román (2005).

- En la primera fase (antes de ver el vídeo), se explican los objetivos del tema que se persiguen con cada vídeo y por qué se centra en los conceptos elegidos, los razonamientos seleccionados o los procedimientos marcados como importantes.

- Durante el visionado del vídeo con la incorporación dela función de interactividad, se pretende sustituir la labor de intermediación característica de la enseñanza presencial. También se enfatizan las posibilidades técnicas que ofrece esta herramienta (pausa, avance rápido, retroceso, etc.), sobre todo, para el uso de las grabaciones de clases teóricas y de resolución de ejercicios prácticos.

- En la tercera fase (después del visionado), se pretende comprobar el grado de asimilación cuantitativo y cualitativo de los contenidos tratados y observados a partir de la realización de diversas actividades (pruebas objetivas, pruebas de desarrollo, tareas en grupos, etc.).

Para realizar este estudio, se ha trabajado con una muestra de alrededor de 200 observaciones en cada uno de los tres cursos académicos analizados (20132014, 2014-2015 y 2015-2016). La población, objeto de estudio, fueron los alumnos matriculados en la asignatura "Matemática Financiera" (primer cuatrimestre del segundo curso del Grado en ADE de la Facultad de Ciencias Económicas y Empresariales de la UNED) durante los tres cursos académicos señalados (1.829 estudiantes en el curso académico 2013-2014, 2.297 en el curso 2014-2015 y 2.093 en el curso 2015-2016). Eso supone que la muestra, con la que se ha trabajado, tiene una buena representatividad puesto que, en el primer curso, supuso el $11 \%$ de la población, en el segundo bajó un poco hasta alcanzar el 8,7\% y, en el tercer año, se situó en el 9,55\%.

$\mathrm{Al}$ objeto de recoger los datos necesarios se elaboró un cuestionario específico para la investigación compuesto por 46 preguntas adaptadas parcialmente de otros cuestionarios extraídos de la literatura científica sobre el tema objeto de estudio (Dankor, 2011; King y He, 2006; Legris, Ingram y Collerette, 2003; Turner et al., 2010) y dividido en cuatro bloques: uno compuesto por 10 preguntas de tipo sociodemográfico (sexo, edad, titulación de acceso, titulación alternativa, ocupación laboral, acceso a internet, horas de estudio semanales, horas de trabajo semanales, nivel previo de matemáticas y número de créditos matriculados) y los otros tres, de 12 preguntas cada uno, relativas al uso de los recursos multimedia utilizados (vía de acceso, momento del visionado, facilidad en la utilización y en la localización, diseño de la presentación, interés didáctico, valor añadido, adquisición de habilidades, utilidad en la consecución de objetivos y utilidad global).

El cuestionario se activó para los estudiantes durante los diez días anteriores a la celebración de la prueba presencial escrita en cada curso académico, y para 
incentivar la participación en la encuesta se otorgaron 0,5 puntos que se añadían a la calificación final solo en el caso de que se alcanzara una calificación mínima de 5 puntos en la prueba presencial escrita (en la línea de otras investigaciones, tales como la de Zhang et al., 2006).

Este diseño es razonable para aquellos casos en los que se pregunta por comportamientos o utilidades (Neuman, 2000) y cuya recopilación se realiza en un período de tiempo relativamente corto (Johnson y Christensen, 2008). El concepto de utilidad empleado en el cuestionario está basado en el modelo TAM diseñado por Davis (1993) y Masrom (2007), al describir la utilidad percibida como el grado en que el usuario considera que el uso de la tecnología (en nuestro caso los vídeos utilizados) puede mejorar su rendimiento en el trabajo.

El cuestionario se puso a disposición de los alumnos a través de la plataforma Google Drive por su facilidad de administración, cuantificación y evaluación por parte del encuestador y por su sencillez de uso para el encuestado. La validez del cuestionario aplicado a esta investigación se ha realizado a través de la prueba de Alfa-Cronbach que analiza si los ítems utilizados, de acuerdo con una escala Likert, están correlacionados (Ritter, 2010). Los valores del coeficiente alfa basado en elementos estandarizados se presentan en la siguiente tabla.

Tabla 1. Validez del cuestionario

\begin{tabular}{|c|c|}
\hline Año académico & Coeficiente alfa \\
\hline $2013-2014$ & 0,960 \\
\hline $2014-2015$ & 0,945 \\
\hline $2015-2016$ & 0,963 \\
\hline
\end{tabular}

Los valores de los coeficientes alfa muy próximos a la unidad indican la consistencia de los ítems seleccionados y, por tanto, la validez del cuestionario realizado.

A partir de los datos obtenidos a través de la encuesta, se generó la correspondiente base de datos que se analizó con el paquete estadístico SPSS 22. Una vez revisada y refinada la matriz de datos, se utilizaron dos tratamientos estadísticos: uno puramente de estadística descriptiva y otro de regresión lineal.

\section{RESULTADOS}

En primer lugar, se analizan y se comparan con estadísticos de naturaleza descriptiva las calificaciones obtenidas en la prueba presencial escrita (febrero o septiembre) por parte de los alumnos, que utilizaron los recursos multimedia y las de aquellos que eligieron no hacer uso de ellos. La media aritmética de las calificaciones para los estudiantes que aprovecharon tales recursos fue de 4,87 puntos para el curso 
académico 2013-2014, de 5,04 puntos para el curso 2014-2015 y de 4,62 puntos para el último curso analizado 2015-2016. En el caso de los estudiantes que no hicieron uso de los recursos multimedia, esas medias aritméticas fueron de 3,55, 3,97 y 3,35 en cada uno de los cursos analizados, respectivamente. Un análisis más completo de las calificaciones por tramos de evaluación se ofrece en la siguiente tabla.

Tabla 2. Comparativa de calificaciones

\begin{tabular}{|l|c|c|l|c|c|c|}
\hline $\begin{array}{c}\text { Uso de } \\
\text { recursos } \\
\text { multimedia }\end{array}$ & Número & Porcentaje & $\begin{array}{c}\text { No uso de } \\
\text { recursos } \\
\text { multimedia }\end{array}$ & Número & Porcentaje \\
\hline \multicolumn{7}{|c|}{ Curso 2013-2014 } \\
\hline Suspensos & 72 & 39,13 & Suspensos & 367 & 57,52 \\
\hline Aprobados & 78 & 42,39 & Aprobados & 212 & 33,23 \\
\hline Notables & 24 & 13,04 & Notables & 45 & 7,05 \\
\hline Sobresalientes & 10 & 5,43 & Sobresalientes & 14 & 2,19 \\
\hline \multicolumn{7}{|c|}{ Curso 2014-2015 } \\
\hline Suspensos & 74 & 36,82 & Suspensos & 502 & 54,68 \\
\hline Aprobados & 83 & 41,29 & Aprobados & 276 & 30,07 \\
\hline Notables & 34 & 16,92 & Notables & 106 & 11,55 \\
\hline Sobresalientes & 11 & 5,47 & Sobresalientes & 34 & 3,70 \\
\hline \multicolumn{7}{|c|}{ Curso 2015-2016 } \\
\hline Suspensos & 88 & 42,51 & Suspensos & 460 & 64,70 \\
\hline Aprobados & 80 & 38,65 & Aprobados & 203 & 28,55 \\
\hline Notables & 37 & 17,87 & Notables & 41 & 5,77 \\
\hline Sobresalientes & 3 & 1,45 & Sobresalientes & 7 & 0,98 \\
\hline
\end{tabular}

En segundo lugar, utilizamos la técnica de regresión lineal múltiple para analizar la relación que existe entre el rendimiento académico (variable dependiente) y la utilidad global que proporcionan los mini videos interactivos y las grabaciones de clases teóricas y prácticas (variables independientes o predictoras). Estableceremos como hipótesis nula que existe independencia lineal entre la calificación global del estudiante en la prueba presencial escrita $(\mathrm{Y})$ y las variables predictoras $\left(\mathrm{X}_{\mathrm{i}}\right)$ que relacionamos a través del siguiente modelo lineal:

$$
\mathrm{Y}_{\mathrm{t}}=\beta_{\mathrm{o}}+\beta_{1} \cdot \mathrm{X} 1+\beta_{2} \cdot \mathrm{X}_{2}+\beta_{3} \cdot \mathrm{X}_{3}+\varepsilon
$$

Las variables predictoras se han ponderado a través de una escala Likert entre 1 y 5 , siendo 1 el valor de utilidad más bajo y 5 el valor de utilidad más alto. El rango del rendimiento académico (variable dependiente) oscila entre la puntuación o (calificación más baja) y la puntuación 10 (calificación más alta). 
Las estimaciones de los coeficientes estandarizados para el modelo anterior se resumen en la siguiente tabla:

Tabla 3. Coeficientes estandarizados beta

\begin{tabular}{|l|c|c|c|}
\hline & Curso 2013-14 & Curso 2014-15 & Curso 2015-16 \\
\hline $\begin{array}{l}\text { Utilidad global mini videos } \\
\text { interactivos }\end{array}$ & 0,003 & 0,125 & 0,144 \\
\hline $\begin{array}{l}\text { Utilidad global grabaciones } \\
\text { clases teóricas }\end{array}$ & 0,068 & 0,186 & 0,029 \\
\hline $\begin{array}{l}\text { Utilidad global grabaciones } \\
\text { clases prácticas }\end{array}$ & 0,186 & 0,014 & 0,087 \\
\hline
\end{tabular}

Teniendo en cuenta que cuanto mayor sea el coeficiente beta (en valor absoluto) más importancia tendrá la variable correspondiente, podemos concluir que, en el primer curso académico del estudio, tuvo más influencia la utilidad global de las grabaciones de las clases prácticas; en el segundo curso académico fueron las grabaciones de las clases teóricas y, en el último curso académico, fueron los mini videos interactivos los que más éxito tuvieron.

La dependencia lineal entre las variables (a través del coeficiente $R^{2}$ ) y el estadístico F de Snedecor se ofrece en la siguiente tabla.

Tabla 4. Cuantificación de la dependencia lineal y estadístico F Snedecor

\begin{tabular}{|c|c|c|c|c|c|c|}
\hline $\begin{array}{c}\text { Curso } \\
\text { académico }\end{array}$ & $\mathrm{R}$ & $\mathrm{R}^{2}$ & $\mathrm{R}^{2}$ ajustado & $\begin{array}{c}\text { Durbin- } \\
\text { Watson }\end{array}$ & $\mathrm{F}$ & Sigma \\
\hline $2013-14$ & 0,262 & 0,069 & 0,053 & 2,043 & 4,409 & 0,005 \\
\hline $2014-15$ & 0,254 & 0,065 & 0,050 & 2,226 & 4,516 & 0,004 \\
\hline $2015-16$ & 0,223 & 0,05 & 0,036 & 1,912 & 3,556 & 0,015 \\
\hline
\end{tabular}

La variable $\mathrm{R}^{2}$ nos indica que las tres variables independientes del modelo explican la calificación obtenida por el alumno en un 6,9\% el primer año, descendiendo ligeramente en el segundo y alcanzando el nivel del $5 \%$ en el último año. En lo que se refiere al parámetro de Durbin-Watson, observamos que los residuos son independientes ya que, en todos los cursos analizados, el valor obtenido se sitúa entre 1,5 y 2,5 (la independencia de los residuos es uno de los supuestos básicos del modelo de regresión lineal).

La hipótesis nula, que se pretende contrastar, se analiza a través de un modelo ANOVA, calculando el estadístico de contraste F y Sigma. Dado que el valor de sigma en todos los años está por debajo de 0,05, se puede rechazar la hipótesis nula de independencia entre el rendimiento académico y las variables independientes: 
utilidad de los mini videos interactivos, utilidad de las grabaciones de clases teóricas y utilidad de las grabaciones de clases prácticas.

La tercera relación analizada es entre la variable dependiente rendimiento académico y las variables independientes horas de estudio, horas de trabajo, nivel de conocimiento de matemáticas comunes, consulta de recursos multimedia ( $\sin$ distinguir entre los tres que se pusieron en el curso virtual).

Para la variable horas de estudio semanales, la ponderación ha sido de 1 para el intervalo o-5 horas, de 2 para el intervalo 6-10 horas y 3 cuando se estudiaban más de 10 horas a la semana. Para la variable horas de trabajo semanales, se ha ponderado con valor 1 si la ocupación laboral suponía menos de 15 horas, con valor 2 para el intervalo 16-25, con valor 3 para el intervalo 26-40 horas y con valor 4 si se trabajaba más de 40 horas a la semana. La tercera variable independiente (nivel de matemáticas) se ha ponderado con valor 1 si se declaraba un nivel de conocimientos inferior a 5, con valor 2 si el nivel declarado se situaba entre 5 y 7 y un valor de 3 para el intervalo 7-10. Por último, la consulta de los recursos multimedia se ha ponderado con valor 1 si se consultaban y o si no se consultaban. En cuanto al rendimiento académico, la ponderación se ha realizado asignando un valor o a la calificación más baja y un valor 10 a la calificación más alta.

El cálculo de los coeficientes estandarizados ilustra la importancia que tiene cada una de las variables a la hora de explicar la variable dependiente.

Tabla 5. Coeficientes estandarizados beta

\begin{tabular}{|l|c|c|c|}
\hline & Curso 2013-14 & Curso 2014-15 & Curso 2015-16 \\
\hline Horas de estudio semanales & 0,016 & 0,014 & 0,098 \\
\hline Horas de trabajo semanales & 0,041 & 0,055 & 0,067 \\
\hline $\begin{array}{l}\text { Nivel previo de matemáticas } \\
\text { comunes }\end{array}$ & 0,389 & 0,285 & 0,284 \\
\hline $\begin{array}{l}\text { Consulta de recursos } \\
\text { multimedia }\end{array}$ & 0,078 & 0,051 & 0,029 \\
\hline
\end{tabular}

Se observa que, a lo largo de toda la serie temporal la variable 'nivel de matemáticas comunes' es la que tiene más influencia en la calificación del alumno a lo largo de toda la serie temporal. 
Tabla 6. Cuantificación de la dependencia lineal y estadístico F Snedecor

\begin{tabular}{|c|c|c|c|c|c|c|}
\hline $\begin{array}{c}\text { Curso } \\
\text { académico }\end{array}$ & $\mathrm{R}$ & $\mathrm{R}^{2}$ & $\mathrm{R}^{2}$ ajustado & $\begin{array}{c}\text { Durbin- } \\
\text { Watson }\end{array}$ & $\mathrm{F}$ & Sigma \\
\hline $2013-14$ & 0,414 & 0,171 & 0,153 & 2,140 & 9,233 & 0,000 \\
\hline $2014-15$ & 0,293 & 0,086 & 0,067 & 2,147 & 4,613 & 0,001 \\
\hline $2015-16$ & 0,316 & 0,100 & 0,082 & 1,806 & 5,616 & 0,000 \\
\hline
\end{tabular}

El cálculo de $\mathrm{R}^{2}$ indica que las cuatro variables independientes del modelo explican la calificación obtenida por el alumno en un $17,1 \%$ el primer año, descendiendo en el segundo y quedando en un $10 \%$ en el último año. No existe un alto grado de dependencia lineal entre las variables y tampoco los residuos son dependientes.

En cuanto al análisis de contraste para aceptar o rechazar la hipótesis nula de independencia entre las variables, se constata a través del estadístico F y Sigma que el ajuste se encuentra en la zona de rechazo de la hipótesis nula, o lo que es lo mismo, se acepta la dependencia lineal entre la variable dependiente rendimiento académico y las variables independientes analizadas. Alternativamente, este estadístico nos permite verificar el cumplimiento de las hipótesis de normalidad y homocedasticidad.

La cuarta relación que analizamos consiste en comparar el valor añadido que le ha reportado al alumno el estudio de la materia a través de los distintos recursos multimedia y una serie de variables independientes dadas por la facilidad en el uso, la localización del material dentro del curso virtual, su propio diseño técnico y el interés didáctico.

Todas las variables se han cuantificado a través de una escala Likert de 1 a 5 , siendo el valor 1 el más bajo de la escala y 5 el máximo considerado.

Para los distintos recursos multimedia, el ajuste de la ecuación de regresión y la prueba de rechazo de la hipótesis de independencia lineal ofrece los siguientes resultados:

Tabla 7. Cuantificación de la dependencia lineal y estadístico F Snedecor

\begin{tabular}{|l|c|c|c|c|c|c|}
\hline $\begin{array}{c}\text { Curso } \\
\text { académico }\end{array}$ & $\mathbf{R}$ & $\mathbf{R}^{2}$ & $\begin{array}{c}\mathbf{R}^{2} \\
\text { ajustado }\end{array}$ & $\begin{array}{c}\text { Durbin- } \\
\text { Watson }\end{array}$ & $\mathbf{F}$ & Sigma \\
\hline Mini videos \\
\hline $2013-14$ & 0,784 & 0,615 & 0,606 & 1,939 & 71,470 & 0,000 \\
\hline $2014-15$ & 0,728 & 0,530 & 0,521 & 1,850 & 55,569 & 0,000 \\
\hline $2015-16$ & 0,777 & 0,603 & 0,595 & 2,057 & 77,110 & 0,000 \\
\hline Grabaciones tutorías teóricas \\
\hline $2013-14$ & 0,795 & 0,632 & 0,624 & 1,956 & 76,908 & 0,000 \\
\hline $2014-15$ & 0,779 & 0,606 & 0,598 & 2,206 & 75,807 & 0,000 \\
\hline $2015-16$ & 0,823 & 0,677 & 0,670 & 2,027 & 106,152 & 0,000 \\
\hline
\end{tabular}




\begin{tabular}{|l|c|c|c|c|c|c|}
\hline $\begin{array}{c}\text { Curso } \\
\text { académico }\end{array}$ & $\mathbf{R}$ & $\mathbf{R}^{2}$ & $\begin{array}{c}\mathbf{R}^{2} \\
\text { ajustado }\end{array}$ & $\begin{array}{c}\text { Durbin- } \\
\text { Watson }\end{array}$ & $\mathbf{F}$ & Sigma \\
\hline Grabaciones clases prácticas \\
\hline $2013-14$ & 0,804 & 0,646 & 0,638 & 2,072 & 81,636 & 0,000 \\
\hline $2014-15$ & 0,848 & 0,719 & 0,714 & 2,020 & 126,299 & 0,000 \\
\hline $2015-16$ & 0,912 & 0,831 & 0,828 & 2,079 & 248,843 & 0,000 \\
\hline
\end{tabular}

Los resultados son bastante ilustrativos de la notable relación que existe para todos los cursos académicos analizados entre los cambios producidos en la variable 'valor añadido al estudio de la materia' a través de los mini vídeos interactivos, las grabaciones de clases teóricas y prácticas y las cuatro variables consideradas como independientes.

\section{DISCUSIÓN Y CONCLUSIONES}

El análisis de los resultados académicos a través de medidas estadísticas de tipo descriptivo muestra que los estudiantes que accedieron a los distintos recursos multimedia (mini videos interactivos, grabaciones de tutorías de tipo teórico y grabaciones de clases prácticas) obtuvieron durante los tres cursos académicos analizados mejores calificaciones que aquellos alumnos que no lo hicieron. Esa mejora del rendimiento se tradujo en una calificación media más alta, en un mayor porcentaje de aprobados y un menor número de suspensos. Además, entre los aprobados se obtuvieron mejores calificaciones en los distintos tramos evaluativos.

Un análisis estadístico más completo examinó la relación entre el rendimiento académico medido por la calificación obtenida en la prueba presencial y la utilidad global que proporcionó a los estudiantes el uso de los recursos multimedia. El resultado de ese análisis fue el rechazo de la hipótesis nula de independencia lineal entre las variables analizadas y la evidencia de que, en el primer curso analizado, las grabaciones de las clases prácticas fueron las de mayor peso, posiblemente por el carácter esencialmente práctico de la materia y la ayuda que les proporcionaba este tipo de material, inédito hasta el momento. En el segundo curso, fueron las grabaciones de las sesiones de tipo teórico las que mejor valoración tuvieron y, en el tercer curso, el protagonismo se lo llevaron los mini vídeos interactivos, probablemente por las mejoras que tuvieron, tanto en la calidad docente como tecnológica, y por la incorporación de la interactividad.

Se demostró también la dependencia lineal entre el rendimiento académico y una serie de variables independientes relacionadas con los hábitos de estudio, entre las que sobresalió el nivel previo de conocimientos matemáticos que tenían los alumnos frente a otras variables consideradas, como el tiempo dedicado al estudio o las horas de trabajo. 
Por último, se evidenció la notable dependencia que existía entre una serie de variables independientes relacionadas con la usabilidad y el diseño de los recursos multimedia y el valor añadido que les proporcionó a los alumnos utilizar estos recursos multimedia, especialmente las grabaciones de las clases prácticas.

Por tanto, los resultados de este estudio empírico realizado durante tres cursos académicos consecutivos y con una muestra representativa de alrededor de 200 alumnos por curso contribuye a consolidar la hipótesis principal que se pretendía contrastar: el uso del vídeo en distintos formatos contribuye positiva y significativamente al aprendizaje de materias de tipo cuantitativo en un entorno de enseñanza universitaria a distancia.

\section{REFERENCIAS BIBLIOGRÁFICAS}

Al-Bahrani, A., y Darshak, P. (2015). Incorporating Twitter, Instagram, and Facebook in Economics Classrooms. The Journal of Economic Education, 46(1), 56-67. doi: http://dx.doi.org/10.1080/o0 220485.2014.978922

Bennett, P., y Glover, P. (2008). Video streaming: Implementation and evaluation in an undergraduate nursing program. Nurse Education Today, 28(2), 253-258. doi:http://dx.doi.org/10.1016/i. nedt.2007.04.005

Berk, R. (2009). Multimedia teaching with video clips: TV, movies, YouTube, and mtvU in the college classroom. International Journal of Technology in Teaching and Learning, 5(1), 1-21. Recuperado de http://goo.gl/U6f3dB

Borup, J., West, R., y Graham, C. (2013). The influence of asynchronous video communication on learner social presence: a narrative analysis of four cases. Distance Education, 34(1), 48-63. doi: http://dx.doi.org/10.1080/01587919 .2013 .770427

Boster, F. J., Meyer, G. S., Roberto, A. J., Inge, C., y Strome, R. (2006). Some effects of video streaming on educational achievement. Communication Education, 55(1), 46-62. doi: http://dx.doi. org/10.1080/03634520500343392
Boster, F. J., Meyer, G. S., Roberto, A. J., Lindsey, L., Smith, R., Inge, C., $y$ Strom, R. (2007). The impact of video streaming on mathematics performance. Communication Education. 56(2), 134-144. doi: http://dx.doi. org/10.1080/03634520601071801

Bravo, E., Amante-García, B., Simo, P., Enache, M., y Fernández, V. (2011). Video as a new teaching tool to increase student motivation. In Proceedings of 2011 IEEE Global Engineering Education Conference, 638-642.

Cabero, J., Llorente, C., y Román, P. (2007). La tecnología cambió los escenarios: el efecto Pigmalión se hizo realidad. Comunicar, 28, 167-175.

Cabero, J., Llorente, C., y Román, P. (2005). Las posibilidades del vídeo digital para la formación. Labor Docente, 4. 58-74. Recuperado de http://goo. gl/7ypjadcontent copy o http:// tecnologiaedu.us.es/cuestionario/ bibliovir/jca9.pdf

Castaño-Muñoz, J., Durant, J., y Teresa, S. (2015). Determinants of internet use for interactive learning: an exploratory study. Journal of New Approaches in Educational Research, 4(1), 25-34.

Cotti, C., y Marianne J. (2012). Teaching economics using historical novels: Jonathan Harr's The Lost Painting. The 
Journal of Economic Education, 43(3), 269-281. http://dx.doi.org/10.1080/002 20485.2012.686391

Donkor, F. (2011). Assessment of learner acceptance and satisfaction with video based instructional method for teaching practical skills at a distance. The International Review of Research in Open and Distance Learning, 12(5), 7492. Recuperado de http://goo.gl/hVoo

Davis, F. D. (1993). User acceptance of information technology: System characteristics, user perceptions, and behavioral impacts. International Journal of Man-Machine Studies, 38, 475-487. Recuperado de http://goo.gl/89JrjE

Dickie, J. (2009). Acknowledging the 'forgotten' and the 'unknown': The role of video podcasts for supporting field-based learning. Planet, 22(1), 6163. doi: http://dx.doi.org/10.11120/ plan.2009.00220061

Dupagne, M., Millette, D. M., y Grinfeder, K. (2009). Effectiveness of video podcast use as a revision tool. Journalism \& Mass Communication Educator, 64(1), 54-70. doi: http://dx.doi. org/10.1177/107769580906400105

Ellis, R., y Childs, M. (1999). The effectiveness of video as a learning tool in online multimedia resources. Journal of Education Media, 24(3), 217-223. doi: 10.1080/1358165990240305.

Estevez, J., y García, A. (2015). Las redes sociales para la mejora de la capacidad de emprender y de autoempleo. International Journal of Education Research and Innovation, 4, 101-110.

Griffin, D. K., Mitchell, D., y Thompson, S. J. (2009). Podcasting by synchronizing PowerPoint and voice: What are the pedagogical benefits? Computers \& Education, 53(2), 532-539. doi: http://dx.doi.org/doi:10.1016/j. compedu.2009.03.011

Hall, J. (2005). Homer economicus: using The Simpsons to teach economics.
Journal of Private Enterprise, 20(2), 166177. Recuperado de http://goo.gl/buu1se Hedge, S., Useem, A., y Martínez, S. (2011). Engaging with business learning: the source and medium do matter. Whitepaper prepared for Big Think, May 25, 2911. Recuperado de http://goo.gl/ $\mathrm{YCMvf3}$

Hee, J. C., y Scott D. J. (2005). The Effect of Context-Based Video Instruction on Learning and Motivation in Online Courses. American Journal of Distance Education, 19(4), 215227. doi: http://dx.doi.org/10.1207 15389286ajde1904 3

Hilgenberg, C., y Tolone, W. (2000). Student perceptions of satisfaction and opportunities for critical thinking in distance education by interactive video. American Journal of Distance Education, 14(3), 59-73. doi: http://dx.doi. org/10.1080/08923640009527065

Hill, J. L., y Nelson, A. (2011). New technology, new pedagogy? Employing video podcasts in learning and teaching about exotic ecosystems. Environmental Education Research, 17(3), 393-408. doi: http://dx.doi.org/10(1080/13504622),20 10,545873

Holtzblatt, M., y Tschakart, N. (2011). Expanding your accounting classroom with digital video technology. Journal of Accounting Education, 29, 100121. doi: http://dx.doi.org/10.1016/j. jaccedu.2011.10.003

Hsin, W. J., y Cigas, J. (2013). Short videos improve student learning in online education. Journal of Computing Sciences in Colleges, 28(5), 253-259. Recuperado de http://goo.gl/GoFxkm

Imbernón, F., Silva, P., y Guzmán, C. (2011). Competencias en los procesos de enseñanza-aprendizaje virtual y semipresencial [Teaching Skills in Virtual and Blenden Learning Environments]. Comunicar, 36, 107-114. doi: http:/ dx.doi.org/10.3916/C36-2011-03-01 
Johnson, R. B., y Christensen, L. B. (2008). Educational research: Quantitative, qualitative, and mixed approaches $\left(3^{\text {rd }}\right.$ ed.). Los Angeles: Sage.

King, W. R., y He, J. (2006). A meta-analysis of the technology acceptance model. Information and Manaqement, 43(6), 740-755. doi:http://dx.doi.org/10.1016/j. im.2006.05.003

Leet, D., y Houser, S. (2003). Economics goes to Hollywood: Using classic films and documentaries to create an undergraduate economics course. The Journal of Economic Education, 34(Fall), 326-332. doi: http://dx.doi. org/10.1080/00220480309595226

Legris, P., Ingham, J., y Collerette, P. (2003). Why do people use information technology? A critical review of the technology acceptance model. Information and Management, 4O(3), 191-204. doi: http://dx.doi.org/10.1016 S0378-7206(01)00143-4

Leijen, A., Lam, I., Wildschut, L., Simons, P. y Admiraal, W. (2008). Streaming video to enhance students reflection in dance education. Computers \& Education, 52(1), 169-176. doi: http://dx.doi.org/10.1016/i. compedu.2008.07.010

Luccasen, R. A., y Thomas, M. K. (2010). Simpsonomics: Teaching economic using episodes of The Simpsons. The Journal of Economic Education, 41(2), 136-149. http://dx.doi. org/10.1080/00220481003613847

Ljubojevic, M., Vaskovic, V., Stankovic, S., y Vaskovic, J. (2014). Using Supplementary Video in Multimedia Instruction as a Teaching Tool to Increase Efficiency of Learning and Quality of Experience. The international review of research in open and distance learning, 15(3), 275-291. Recuperado de http://goo.gl/TJAQVp

Martínez, F. (2004). Bases generals para el diseño, la producción y la evaluación de medios para la formación. En J. A. Salinas
(Coord.), Tecnologías para la educación. Madrid. Alianza.

Masrom, M. (2007). Technology Acceptance Model and E-learning. $12^{\text {th }}$ International Conference on Education. Sultan Hassanal Bolkiah Institute of Education. University Brunei Darussalam. May 2007. Recuperado de http://eprints.utm. my/5482

Mateer, G. D., y Li, H. (2008). Movie scenes for economics. The Journal of Economic Education, 39(3), 303. doi: http://dx.doi. org/10.3200/JECE.39.3.303

Mbarika, V., Sankar, C., Raju, P., y Raymond, J. (2000). Importance of learning-driven constructs on perceived skill development when using multimedia instructional materials. The Journal of Educational Technology Systems. 29(1), 67-87. http:/ dx.doi.org/10.2190/8G4W-2W33-UKUBoLH5

Neuman, W. L. (2000). Social research methods: Qualitative and quantitative approaches ( $4^{\text {th }}$ ed.). Boston: Allyn and Bacon.

O’Bannon, B., Lubke, J., Beard, J., y Britt, V. (2011). Using podcasts to replace lecture: Effects on student achievement. Computers \& Education, 57(3), 18851892. doi: http://dx.doi.org/10.1016/i. compedu.2011.04.001

Pascual, M. A. (2011). Principios pedagógicos en el diseño y producción de nuevos medios, recursos y tecnologías. En M. L. Sevillano (Coord.), Medios, recursos didácticos y tecnología educativa. Madrid: Pearson Educación.

Ritter, N. (2010). Understanding a widely misunderstood statistic: Cronbach's alpha. Paper presented at the annual meeting of the Southwest Educational Research Association. New Orleans.

Rodriguez, C. (2011). Capitalism in six westerns by John Ford. The Journal of Economic Education, 42(2), 191-194. doi: http://dx.doi.org/10.1080/00220485.20 11.555721 
Ross, T. K., y Bell, P. D. (2007). "No significant difference" only on the surface. International Journal of Instructional Technology and Distance Learning, 4(7), 3-13. Recuperado de http://goo.gl/ bj3pDy

Salomon, G. (1979). Interaction of media, cognition and learning. Londres: Jessey Bass.

Salomon, G. (1981). Communication and education: an interactional approach. Los Angeles. Sage.

Sexton, R.L. (2006). Using Short Movie and Television Clips in the Economics Principles Class. The Journal of Economic Education, 37(4), 406-417. doi: http: / dx.doi.org/10.3200/JECE.37.4.406-417

Siegfried, J. R., Bartlett, W., Hansen, W. L., Kelley, A. C., McCloskey, D. N., y Tietenberg, T. H. (1991). The status and prospects of economics majors. The Journal of Economic Education, 22(Summer), 197-224. Recuperado de http://goo.gl/HRhble

Sigüenza, J. (2004). Diseño de materiales docentes multimedia en entornos virtuales de enseñanza-aprendizaje. Recuperado de http://www.ucm.es/info/multidoc/ multidoc/revista/num8/siguenza.html

Stiubiener, I., Silveira, R., Matushima, R., Bressan, G. y Ruggiero, W. (2012). Evaluating the effectiveness of the use of teaching materials in video format in distance learning environments. In Frontiers in Education Conference Proceedings 2012, 1-6.

Tinari, F.D. y Khandke, K. (2000). From Rhytm and blues to Broadway: using music to teach economics. The Journal of Economic Education, 31(3), 253-270. doi: http://dx.doi. org/10.1080/00220480009596784

Turner, M., Kitchenham, B., Brereton, P., Charters, S., y Budgen, D. (2010). Does the technology acceptance model predict actual use? A systematic literature review. Information and Software Technology, 52(5), 463-479. doi: http://dx.doi. org/10.1016/j.infsof.2009.11.005

Wallace, R. (2003). Online learning in higher education: a review of research on interactions among teachers and students. Education, Communication \& Information, 3(2), 241-280. doi: http:// dx.doi.org/10.1080/14636310303143

Watts, M. (2003). The literary book of economics. Wilmington, DE: ISI.

Wieling, M., y Hofman, W. (2010). The impact of online video lecture recordings and automated feedback on student performance. Computers \& Education, 54(4), 992-998. doi: http://dx.doi. org/10.1016/j.compedu.2009.10.002

Vachis, M. A., y Bohanan, C.E. (2012). Using illustrations from American novels to teach about labor market. The Journal of Economic Education, 42(1), 72-82. doi: http://dx.doi.org/10.1080/00220485.20 12.636712

Vajoczki, S., Watt, S., Marquis, N., y Holshausen, K. (2010). Podcasts: Are they an effective tool to enhance student learning? A case study from McMaster University, Hamilton Canada. Journal of Educational Multimedia and Hypermedia, 19(3), 349-362. Recuperado de https://goo.gl/zVhro4

Yanghee, K., y Thayne, J. (2015). Effects of learner-Instructor relationship-building strategies in online video instruction. Distance Education, 36(1), 100-114. doi: http://dx.doi.org/10.1080/01587919.201 5.1019965

Zhang, D., Zhou, L., Briggs, R., y Nunamaker, J. (2006). Instructional video in e-learning: Assesing the impact of interative video on learning effectiveness. Information \& Management, 43(1). 1527. doi: http://dx.doi.org/10.1016/i. im.2005.01.004 


\section{PERFIL ACADÉMICO Y PROFESIONAL DE LOS AUTORES}

Damián de la Fuente Sánchez. Doctor en Ciencias Económicas y Empresariales por la UNED. Profesor Titular de Universidad en el Departamento de Economía de la Empresa y Contabilidad. Ha desempeñado diversos cargos en la UNED, siendo en la actualidad el director del Departamento de Economía de la Empresa y Contabilidad. Ha presentado numerosas comunicaciones en congresos nacionales e internacionales relacionados con la Matemática Financiera y la innovación docente en esta disciplina.

E-mail: dfuente@cee.uned.es

Montserrat Hernández Solís. Doctora en Ciencias Económicas y Empresariales por la Universidad Complutense de Madrid. Profesora Ayudante Doctor en el Departamento de Economía de la Empresa y Contabilidad. Autora de publicaciones relacionadas con los seguros de vida y la utilización de recursos tecnológicos aplicados a la docencia de las finanzas.

E-mail: montserrath@cee.uned.es

Inmaculada Pra Martos. Doctora en Ciencias Económicas y Empresariales por la UNED. Profesora de Economía Financiera en el Departamento de Economía de la Empresa y Contabilidad. Ha desempeñado diversos cargos de gestión en el rectorado y en el decanato de la Facultad de Económicas. Sus principales líneas de investigación y publicación tienen que ver con el riesgo de crédito y supervisión bancaria, microfinanzas, y la aplicación de nuevas tecnologías en la educación superior.

E-mail: ipra@cee.uned.es

\section{DIRECCIÓN DE LOS AUTORES}

Facultad de Ciencias Económicas y Empresariales

Universidad Nacional de Educación a Distancia (UNED)

Paseo Senda del Rey, 11

28.040 Madrid (España)

Fecha de recepción del artículo: 22/02/2017

Fecha de aceptación del artículo: 24/04/2017 


\section{Como citar este artículo:}

De la Fuente Sánchez, D., Hernández Solís, M., y Pra Martos, I. (2018). Vídeo educativo y rendimiento académico en la enseñanza superior a distancia. RIED. Revista Iberoamericana de Educación a Distancia, 21(1), pp. 323-341. doi: http:// dx.doi.org/10.5944/ried.21.1.18326 